Felipe S. Torres

Andrew M. Crean

Narinder Paul

\section{Letter to the Editor: re: Body physique and heart rate variability determine the occurrence of stair-step artefacts in 64-slice CT coronary angiography with prospective ECG-triggering}

Received: 25 March 2009

Accepted: 20 April 2009

Published online: 23 July 2009

(C) European Society of Radiology 2009
The reply to this Letter to the Editor is available at doi:10.1007/s00330-009-1484-5.

\section{F. S. Torres $(\bowtie) \cdot$ A. M. Crean ·}

N. Paul

Division of Cardiothoracic Imaging,

Department of Medical Imaging

Toronto General Hospital,

585 University Ave,

Toronto, ON, Canada

e-mail: felipe.torres@uhn.on.ca

Tel.: +1-416-3404800

Fax: +1-416-3403900
Sir,

We read with great interest the article by Husmann and colleagues entitled "Body physique and heart rate variability determine the occurrence of stair-step artefacts in 64-slice CT coronary angiography with prospective ECG-triggering" [1]. The authors evaluated the presence and the determinants of stair-step artefacts in computed tomography coronary angiography (CTCA) with prospective ECG-triggering performed using 64slice multidetector CT (64-MDCT).

In order to separate the effects of respiratory motion on the prevalence of stair-step artefacts, 13 patients were not included in the study "because they did not follow the breathing commands properly". It is worth noting that "breathing commands were practised repetitively" before CT and that all patients were "carefully monitored during the examination in an effort to assure that breathing commands were adequately followed". Therefore, we may assume that, despite careful coaching, $8.3 \%$ (13/156) of patients presented artefacts due to respiratory motion.

Respiratory motion causes stair-step artefact both in the chest wall and in the coronary arteries [2]. This type of artefact frequently cannot be corrected by postprocessing techniques such as multisegment reconstruction or ECGediting [3], which are both common strategies used to overcome the effects of cardiac motion. Therefore, stair-step artefact due to respiratory motion may render a coronary segment unevaluable. The high incidence of thoracic wall stair-step artefacts (54\%, 77/143) reported is concerning for a significant detrimental effect on vessel evaluability which was not assessed by the authors.

The study by Husmann and colleagues brings to attention two important factors that may impair coronary segments evaluability through stair-step artefacts in prospective ECG-triggered CTCA: body physique and heart rate variability. However, the study indirectly reveals the high frequency of respiratory motion and encourages investigators to study this additional and overlooked source of image degradation in CT coronary angiography.

Declaration of conflict of interest Dr. Narinder Paul has received research support from Toshiba Medical Systems.

\section{References}

1. Husmann L, Herzog BA, Burkhard N et al (2009) Body physique and heart rate variability determine the occurrence of stair-step artefacts in 64-slice CT coronary angiography with prospective ECG-triggering. Eur Radiol. doi:10.1007/s00330-009-1339-0

2. Choi HS, Choi BW, Choe KO et al (2004) Pitfalls, artifacts, and remedies in multi-detector row CT coronary angiography. Radiographics 24 : 787-800

3. Nakanishi T, Kayashima Y, Inoue R, Sumii K, Gomyo Y (2005) Pitfalls in 16-detector row $\mathrm{CT}$ of the coronary arteries. Radiographics 25:425-438 